co-floxing the mTORC1 essential component Raptor. Translational status was assessed by sucrose gradient ultracentrifugation of intestinal epithelial extract from these mice and ${ }^{35} \mathrm{~S}$ methionine incorporation and harringtonine chase assays on organoid cultures. The role of downstream mTORC1 effectors was established by assessing the intestinal regeneration following IR irradiation of $4 \mathrm{EBP} 1 / 2^{\mathrm{DKO}}, \mathrm{S} 6 \mathrm{~K} 1 / 2^{\mathrm{DKO}}, \mathrm{rpS} 6^{\mathrm{mut}}$ and $\mathrm{eEF} 2 \mathrm{k}^{-/-}$mice. Survival studies for Apc ${ }^{\mathrm{fl} / \mathrm{fl}}$ mice treated with rapamycin were performed both prior to, and on development of, symptoms

Results mTORC1 activity is absolutely required for the proliferation of Apc deficient, but not wild type, intestinal crypts. Surprisingly, although protein synthesis is increased in Apc $\mathrm{c}^{\mathrm{f} / \mathrm{fl}} \mathrm{crypts}$, it is translation elongation and not initiation that is the rate limiting step. Mechanistically, the inhibition of eukaryotic elongation factor (eEF2) kinase, to increase eEF2 activity downstream of mTORC1 and S6K is required for Wnt-mediated proliferation after IR irradiation. Treatment of established Apc $\mathrm{fl}^{\mathrm{f} / \mathrm{fl}}$ adenomas with rapamycin (which inhibits the mTORC1-S6K-eEF2k-eEF2 axis) arrests tumour growth and prolongs life. Furthermore, rapamycin treatment of mice immediately following homozygous Apc loss prevents the onset of symptoms.

Conclusion These data show that intestinal adenoma formation and growth requires an mTOR mediated increase in translation elongation. Treatment of patients at high risk of developing CRC, such as those with Familial Adenomatous Polyposis, with Rapalogs may therefore be of therapeutic value.

Disclosure of Interest None Declared.

\section{PWE-022 PATIENT ACCEPTABILITY OF A NOVEL, NON-INVASIVE METHOD OF COLONIC SAMPLING FOR BIOMARKER ANALYSIS}

${ }^{1} \mathrm{~V}$ Chhaya*, ${ }^{1} \mathrm{~A}$ Poullis, ${ }^{2} \mathrm{~T}$ Bandaletova, ${ }^{2} \mathrm{~A}$ Loktionov. ${ }^{1}$ Gastroenterology, St Georges Hospital, London, UK; ${ }^{2}$ DiagNodus Ltd, Cambridge, UK

\subsection{6/gutjnl-2014-307263.282}

Introduction Biomarker analysis is commonly used for the diagnosis of those presenting with colorectal symptoms and monitoring of inflammatory bowel disease (IBD), but the acceptability of stool sampling is poor. It is proposed that material rich in the colonic mucocellular layer is deposited on the anal surface postdefaecation and can be sampled for biomarker analysis. Our aim was to develop a novel, non-invasive method of material collection post-defaecation and assess patient acceptability.

Methods Patients with IBD (active cases and those in remission), irritable bowel syndrome (IBS) and a group of healthy volunteers were recruited. Participants were instructed to collect material from the surface of the anus immediately post-defaecation using a specially designed swab covered with flocked nylon (designed by DiagNodus Ltd). The collection process and preparation of samples was performed by patients at home using a specially designed kit. Samples were mailed back, ready for cytological and immunochemical analysis. Patients were provided with kits

\begin{tabular}{llc} 
Abstract PWE-022 Table 1 & & \\
\hline & Mean & SD \\
\hline Convenience/Acceptability & 4.57 & 0.497 \\
Sampling ease & 4.54 & 0.595 \\
Adequacy of time required for sampling & 4.53 & 0.575 \\
Overall impression & 4.57 & 0.553 \\
\hline
\end{tabular}

and asked to collect and return samples at predefined timepoints. Patients completed a simple questionnaire to evaluate: (a) procedure convenience/acceptability, (b) ease of sample collection, (c) adequacy of time required for sampling and (d) overall impression each using a 5 -point rating scale $(1=$ poor to $5=$ good).

Results 112 patients were recruited comprising of 60 patients with active IBD, 14 patients with IBD in remission, 31 patients with IBS and 7 healthy volunteers. Collected samples were returned by $97(86.6 \%)$ study participants $(88.3 \%$ of patients with active IBD, $78.6 \%$ of patients with IBD in remission, $87.1 \%$ of patients with IBS and $100 \%$ of healthy volunteers). Completed questionnaires were returned by 92 trial participants $(94.8 \%$ of those providing samples). The mean and standard deviation (SD) of participant responses is provided in the table below.

Sampling was not associated with discomfort or harm. The material obtained proved suitable for both cytological assessment and protein biomarker estimation.

Conclusion Material from the colonic mucocellular layer deposited in the anal area following defaecation is readily collectable using our specially designed kit and can provide material for both cytological assessment and biomarker quantification. This simple, reliable process is well tolerated and convenient as patients can provide samples from the comfort of their own home. This new technique warrants further study in different patient groups.

Disclosure of Interest V. Chhaya: None Declared, A. Poullis: None Declared, T. Bandaletova Shareholder of: DiagNodus Ltd, Conflict with: Founder of DiagNodus Ltd, A. Loktionov Shareholder of: DiagNodus Ltd, Conflict with: Founder of DiagNodus Ltd.

\section{PWE-023 IS IT WORTH CHASING INCIDENTAL COLONIC HOT SPOTS ON ROUTINE PET CT SCANS?}

${ }^{1}$ WS Ngu*, ${ }^{2} \mathrm{R}$ Sinha, ${ }^{1} \mathrm{D}$ Aitken, ${ }^{2} \mathrm{~A}$ Ramadas, ${ }^{1} \mathrm{~A}$ Reddy, ${ }^{1} \mathrm{M}$ Jha. ${ }^{1}$ Colorectal Surgery, The James Cook University Hospital, Middlesbrough, UK; ${ }^{2}$ Gastroenterology, The James Cook University Hospital, Middlesbrough, UK

\subsection{6/gutjnl-2014-307263.283}

Introduction Positron emission tomography (PET) measures metabolic changes at a cellular level enabling detection of early stage disease. Incidental 2-deoxy-[ $\left.{ }^{18 \mathrm{~F}} \mathrm{~F}\right]$ fluoro-2-D-glucose (FDG) colonic uptake is detected in $1.3-3 \%$ of patients with up to a third resulting in false positive results. ${ }^{1}$ Follow-up endoscopy is recommended to further distinguish these FDG avid lesions. ${ }^{2}$ Cancer detection rates of $7.8-18.9 \%$ have been quoted in various studies. ${ }^{1,3}$ Our aim was to evaluate colonic FDG avid lesions on PET by endoscopy.

Methods An analysis of prospectively collected database of all patients $(\mathrm{n}=1564)$ who had PET for various malignancy between January 2011 to September 2013 was performed.

Results Fifty-nine (3.77\%) patients had focal colonic FDG uptake and $45(2.87 \%)$ patients went on to have colonoscopy.

Indications for PET CT for those undergoing endoscopy was lung carcinoma (22), gastrointestinal carcinoma (10), laryngeal carcinoma (7) and lymphoma (6).

Median age was 64 with a male preponderance $(2.5: 1)$

Location on PET CT was categorised to sigmoid (23), rectal (9), anorectal (4), caecal (3), hepatic flexure (2), transverse (1), splenic flexure (1), ascending (1) and descending (1).

Findings on endoscopy ranged from polyps (22), normal (9), diverticulosis (8), sigmoid cancer (4), caecal cancer (1) and colitis (1). 